\title{
Molecular based detection, genetic characterization and phylogenetic analysis of porcine circovirus 4 from Korean domestic swine farms
}

\author{
Giap Nguyen ${ }^{1}$, Hai-Quynh Do $^{2}$, Le Huynh ${ }^{1}$, Yong Ho Park ${ }^{3}$, Bong Kyun Park ${ }^{3}$, and \\ Heechun Chung ${ }^{4}$ \\ ${ }^{1}$ Vietnam National University of Agriculture \\ ${ }^{2}$ Affiliation not available \\ ${ }^{3}$ Seoul National University \\ ${ }^{4}$ Seoul Nationational University
}

October 5, 2020

\begin{abstract}
Porcine circovirus 4 (PCV4), a novel and unclassified member of the genus Circovirus, was first reported in China in 2019. Aimed at providing more evidence about the active circulation of PCV4, this study screened 335 pooled internal organs and detected the virus (i) at the rates of $3.28 \%$, (ii) from both clinical healthy and clinical sick pigs of various age groups, and (iii) in six out of nice provinces of Korea. The complete genomic sequence of a Korean PCV4 strain (E115) was 1,770 nucleotides in length and had $98.5 \%$ to $98.9 \%$ identity to three PCV4 strains available at GenBank up to date. Utilizing a set of bioinformatic programs, it was revealed that the Korean PCV4 strain contained several genomic features of (i) a palindrome stem-loop structure with conserved nonanucleotide, (ii) packed overlapping ORFs oriented in different directions, and (iii) two intergenic regions in between genes encoding putative replication- associated protein (Rep) and capsid (Cap) proteins. This study also predicted the presence of essential elements known so far for the replication of circoviruses, for example, the origin of DNA replication, endonuclease and helicase domains of Rep, the nuclear localization signal on the putative Cap protein. Finally, based on the phylogeny inferred from sequences of the putative Rep protein, it was suggested that PCV4 belong to genus Circovirus of family Circoviridae and losely related to three previous known porcine circoviruses of PCV1, PCV2 and PCV3.
\end{abstract}

\section{[Transboundary and Emerging Diseases]}

(Original Article)

Molecular based detection, genetic characterization and phylogenetic analysis of porcine circovirus 4 from Korean domestic swine farms

Author: Van-Giap Nguyen ${ }^{1}$, Hai-Quynh Do ${ }^{2}$, Thi-My-Le Huynh ${ }^{1}$, Yong-Ho Park ${ }^{3}$, Bong-Kyun Park ${ }^{4}$, HeeChun Chung ${ }^{4^{*}}$

Affiliations: ${ }^{1}$ Department of Veterinary Microbiology and Infectious Diseases, Faculty of Veterinary Medicine, Vietnam National University of Agriculture, Hanoi, Vietnam; ${ }^{2}$ Institute of Genome Research, Vietnam Academy of Science and Technology, Hanoi, Vietnam. ${ }^{3}$ Department of Veterinary Microbiology, College of Veterinary Medicine and Research Institute for Veterinary Science, Seoul National University, Seoul 151742, Republic of Korea; ${ }^{4}$ Department of Veterinary Medicine Virology Lab, College of Veterinary Medicine and Research Institute for Veterinary Science, Seoul National University GwanAk-Ro 1, GwanAk-Gu, Seoul 151-742, Korea. 
Correspondences: heeskyi@snu.ac.kr (H.-C.C)

${ }^{*}$ Correspondence : heeskyi@snu.ac.kr (H.-C.C)

Addresses for correspondence : H. C. Chung, Department of Veterinary Medicine Virology Lab, College of Veterinary Medicine and Research Institute for Veterinary Science, Seoul National University DaeHakRo 1, GwanAk-Gu, Seoul 151-742, Korea.

Tel.: +82-2-880-1255, Fax: +82-2-885-0263

Running title : Identification of porcine circovirus 4 in Korea

\section{Summary}

Porcine circovirus 4(PCV4), a novel and unclassified member of the genus Circovirus, was first reported in China in 2019. Aimed at providing more evidence about the active circulation of PCV4, this study screened 335 pooled internal organs and detected the virus (i) at the rates of $3.28 \%$, (ii) from both clinical healthy and clinical sick pigs of various age groups, and (iii) in six out of nice provinces of Korea. The complete genomic sequence of a Korean PCV4 strain (E115) was 1,770 nucleotides in length and had 98.5\% to 98.9\% identity to three PCV4 strains available at GenBank up to date. Utilizing a set of bioinformatic programs, it was revealed that the Korean PCV4 strain contained several genomic features of (i) a palindrome stem-loop structure with conserved nonanucleotide, (ii) packed overlapping ORFs oriented in different directions, and (iii) two intergenic regions in between genes encoding putative replication- associated protein (Rep) and capsid (Cap) proteins. This study also predicted the presence of essential elements known so far for the replication of circoviruses, for example, the origin of DNA replication, endonuclease and helicase domains of Rep, the nuclear localization signal on the putative Cap protein. Finally, based on the phylogeny inferred from sequences of the putative Rep protein, it was suggested that PCV4 belong to genus Circovirus of familyCircoviridae and losely related to three previous known porcine circoviruses of PCV1, PCV2 and PCV3.

Keywords: porcine circovirus 4, detection, genome organisation, phylogenetic analysis

\section{Introduction}

With the explosion of sequencing technology and development of bioinformatic tools, an eruption in the number of novel microorganisms, especially viruses, were discovered in a variety of sample types (Mokili, Rohwer, \& Dutilh, 2012; Tisza et al., 2020). In the field of veterinary medicine, since the detection of a closed circular single-stranded DNA virus (porcine circovirus, PCV) (Tischer, Gelderblom, Vettermann, \& Koch, 1982), four circular DNA viruses were detected from clinically diseased pigs, including PCV2 (Meehan et al., 1998), P1 (Wen et al., 2012), PCV3 (Palinski et al., 2017) and the most recent PCV4 (Zhang et al., 2020). Up to date, by reverse genetics, the genomic clones of PCV2, P1 and PCV3 were demonstrated to be infectious and were able to induce gross and histopathological lesions in experiment specific pathogen free or conventional pigs (Fenaux et al., 2002; Jiang et al., 2019; Wen et al., 2012). Despite that, only PCV2 is widely acknowledged as an primary causative agent of porcine circovirus-associated diseases (Opriessnig, Meng, \& Halbur, 2007; Segales, 2012).

Of the 2 viruses most recently described, PCV3 and PCV4 viruses, PCV3 was widely detected in Asia (Kedkovid et al., 2018; D. Zhao et al., 2018), Europe (Faccini et al., 2017; Franzo et al., 2018) and America (Arruda et al., 2019; Tochetto et al., 2018). In contrast, to date, PCV4 were reported in China (Tian et al., 2020; Zhang et al., 2020) but none were detected in retrospective samples collected from 1997- 2018 in Spain and Italy (Franzo et al., 2020). In order to contribute to the understanding of geographic distribution and genomic structure of this novel virus, this study firstly performed molecular based screening for the presence of PCV4 in Korea from tissue samples collected from August 2019 to August 2020. Upon having complete genomic sequence of PCV4, a wide range of bioinformatic tools were utilized to investigate the potential biological important proteins encoded by PCV4 which might play roles in the replication process and/or pathogenesis of PCV4 in pigs. 


\section{Materials \& Method}

2.1. Sample collection and nucleic acid extraction

The screening for PCV4 was done on 335 samples which were divided into six groups including aborted fetuses $(\mathrm{n}=86)$, suckling $(\mathrm{n}=43)$, weaner $(\mathrm{n}=135)$, grower $(\mathrm{n}=23)$, finisher $(\mathrm{n}=20)$ and sow $(\mathrm{n}=28)$. Except testicles of healthy suckling pigs, which were submitted for herd monitoring of PRRSV, the other pooled internal organs (lungs, spleen, heart, kidneys) of dead pigs/ aborted fetuses were sent for detection of common pathogens of swine. The sampling period was from August- December 2019 and January- August 2020, and covered 36 farms in nine provinces in South Korea. The samples were homogenized, diluted 10fold with phosphate-buffered saline (PBS 0.1M, pH 7.4), then stored at -70. The extraction of total nucleic acid was done using DNA/RNA extraction kit (iNtRON Biotechnology, Inc. Korea). Afterward, extracted nucleic acid samples were again stored at -70 .

\subsection{The detection of PCV4}

The detection of PCV4 was based on PCR assay utilizing specific primers designed in the previous study (Tian et al., 2020). The upstream primer (PCV4F) was 5'-GTTTTTCCCTTCCCCCACATAG3', located at nucleotide (nt) 1347-1368, and the downstream primer (PCV4R) was 5'ACAGATGCCAATCAGATCTAGGTAC-3', located at nt 1713-1737. All primers used in this study were synthesized by Macrogen Co., Ltd., Korea.

The PCR reaction mixture consisted of $2 \mu \mathrm{L}$ of template DNA, $1 \mu \mathrm{L}$ of each primer $(10 \mu \mathrm{M})$ and $16 \mu \mathrm{L}$ of Master mix solution (iNtRON Biotechnology, Inc. Korea). The PCR thermal profile was an initial denaturation for 5 min at 95, followed by 40 cycles of 95 for $30 \mathrm{sec}, 58$ for $30 \mathrm{sec}, 72$ for $30 \mathrm{sec}$, and a final extension for 7 min at 72 . The PCR products were analyzed through electrophoresis on $1 \%$ agarose gel with Red Safe DNA nucleic acid staining solution (iNtRON Biotechnology, Inc. Korea).

\subsection{Complete genome amplification and sequencing}

We amplified the complete PCV4 genome from the positive samples. For the genomic sequence analysis, two pairs of primers (first forward, 5'-CCGTGAGTTCCCGTCTGTAT-3', located at nt 464-483; first reverse, 5'-TGGAGACTATGTGGGGGAAG-3', located at nt 1355-1374; second forward, 5'-GCTGGAAGTGGAGGGTGTG-3', located at nt 1220-1238; second reverse, 5'CCCCTTCTCTTGTACATGTCT-3', located at nt 600-620) were designed in this study. The designed primer sequences are based on the genomic sequence of PCV4 strain HNU-AHG1-2019 (accession number: MK986820). The PCR thermal profile was initially denatured for 5 min at 95, followed by 40 cycles of 30 $\mathrm{sec}$ at 95, $2 \mathrm{~min}$ at 55, $1 \mathrm{~min} 20 \mathrm{sec}$ at 72 for, and final extension for $7 \mathrm{~min}$ at 72 . After screening, positive samples were sent to a commercial facility (Macrogen Co., Ltd. Korea) for sequencing.

\subsection{Genomic characterization of PCV4}

The putative ORFs of E115 strain (GenBank no. MT882344) and the other three PCV4 genomes available from GenBank (MT015686, MK986820, MK948416) were predicted using ORF Finder tool (https://www.ncbi.nlm.nih.gov/orffinder/, last accessed on August 2020) with the minimum length of 50 amino acids and the start codon was strictly selected as ATG. The function of the putative proteins of PCV4 were analyzed by BLAST (Johnson et al., 2008) and InterPro (Mitchell et al., 2019). Other motifs appeared in the putative encoding genes and were identified using ScanProsite (de Castro et al., 2006), using the PROSITE databases and other publication functional motifs, reviewed by Sobhy (2016). The putative nuclear localization signal (NLS) was predicted by cNLS Mapper (Kosugi, Hasebe, Tomita, \& Yanagawa, 2009).

Sequence alignment was done by MAFFT using the L-INS-i method (Katoh \& Standley, 2013). Conserved regions from aligned sequences were further analyzed and represented by MEME-suit programs (Bailey et al., 2009). The conserved motifs were identified as short peptides with at least 4 residues in length which were mostly unchanged among input sequences or substituted by ones with similar biochemical properties. 
The protein-peptide interaction function of the conserved motifs was anticipated by LMDIPred web server which predicts short linear peptides that might bind to SH3, WW or PDZ modules (Sarkar, Jana, \& Saha, 2018).

\subsection{Phylogenetic analyses}

Because of its novelty, the taxonomy status of PCV4 has not been determined. Additionally, with the extreme diversity of CRESS DNA viruses (a group of circular, single-stranded DNA viruses encoding replicationassociated protein) (Rosario, Duffy, \& Breitbart, 2012; L. Zhao, Rosario, Breitbart, \& Duffy, 2019), it was our curiosity to have a broad visualization of the genetic relationship of PCV4 with others CRESS DNA viruses. As a result, the phylogenetic analyses were done based on amino acid alignments of replicationassociated protein (Rep), utilizing reference sequences described in the previous studies (Kazlauskas, Varsani, \& Krupovic, 2018; Krupovic et al., 2020; Rosario et al., 2017). The alignment of the putative Rep sequences was done by COBALT tool (Papadopoulos \& Agarwala, 2007). Complete lists of Rep sequences were given in Supplementary data S1- S2.

Using IQ-TREE v2.1.1 (Minh et al., 2020), the genetic relationships between CRESS DNA viruses were inferred by maximum likelihood method. The '-m MFP' option was invoked which helps selecting the data best-fit amino acid substitution model. The branch support values were estimated by ultrafast bootstrap approximation (Hoang, Chernomor, von Haeseler, Minh, \& Vinh, 2018) via "-bb 1000" option. All phylogenies were midpoint rooted and displayed by FigTree v1.4.3 (http://tree.bio.ed.ac.uk/software/figtree/).

\section{Results}

\subsection{PCR- based detection of PCV4}

Among 335 samples, 11 (3.28\%) were positive using PCV4F and PCV4R. Though the detection rates seemed quite low, two notices were obvious. It was the wide distribution of PCV4 in six out of nine sampling sites (Figures 1A-1B). And the virus appeared to be present in all age groups and aborted fetuses (Figure 1C). There were no PCV4 positive samples in the finisher group, which was highly likely due to small sample size. Noteworthy, PCV4 was both found in clinically healthy (testicles of piglets) and clinical sick pigs (pooled internal organs).

\subsection{Genome organization and viral proteins functional analysis}

The full genome of a Korean PCV4 strain (E115) was 1,770 nucleotides in length, showing 98.5\% to 98.9\% similarity to three PCV4 strains available at GenBank (MT015686, MK986820, MK948416). Based on the ORFfinder results, the genome of E115 strain generated in this study was predicted to contain two common ORFs encoding for putative replication-associated protein (Rep) and putative capsid (Cap) protein and four other short ORFs oriented in the different directions (Figure 2A). All of the putative ORFs identified in E115 strain were also detected in the genome of previous known PCV4 strains (Supplementary Figure S1). A palindrome stem-loop structure with the conserved nonanucleotide (CAGTATTAC) was observed (Figures 2B- 2C). In detail, a 11- nucleotide of loop structure was flanked by two reversed complement regions of 16 nucleotides each (Figure 2B). Additionally, the putative Rep and Cap genes were separated by two intergenic regions of 111 and 81 nucleotides in length at the 5' and 3', respectively (Figure 2A and Supplementary Table S1).

Further analysis results (Figure 3) indicated that the putative Rep of E115 strain contained (i) the endonuclease domain with three motifs, (ii) the helicase domain of superfamily 3 (SF3) containing three Walker motifs and (iii) other unknown conserved motifs which are normally observed in Circoviridae in particular and in CRESS DNA viruses in general as previously described (Delwart \& Li, 2012; Kazlauskas et al., 2018; Krupovic et al., 2020; Ye, Berg, Fossum, Wallgren, \& Blomstrom, 2018).

Of the putative Cap, a nuclear localization signal (NLS) was predicted in the N-terminus of the putative Cap of E115 strain (Figure 4). Based on the alignment with other members of Circovirus genus, Figure 4 showed that the arginine-rich region of E115 strain was aligned with the basic motifs of the experimentally confirmed 
NLS of PCV1, PCV2 and PCV3 (Liu, Tikoo, \& Babiuk, 2001; Mou, Wang, Pan, \& Chen, 2019; Shuai et al., 2008). Additionally, motif screening (Figure 5) indicated that the putative Cap of E115 strain contained several tyrosine-based $\mathrm{Y}-\mathrm{x}-\mathrm{x}-\varphi$ motifs and $\mathrm{P}-\mathrm{x}-\mathrm{x}-\mathrm{P}$ motifs (x represents any amino acid and $\varphi$ denotes a large hydrophobic residue of either F, I, L or V) which were related to clathrin-mediated endocytosis (Sobhy, 2016).

Of the other four putative ORF3- ORF6 of E115 strain, only ORF3 protein contained homologous regions with a recently identified ORF5 protein of PCV2 (Lv, Guo, Xu, Wang, \& Zhang, 2015). Widely comparing with homologous putative proteins of some members of genus Circovirus, it was found that they shared some short, conserved linear peptides of W-A-S-[PL]-[DG]-M, G-G-M-x-[TI], W-M-[TI]-[IF]-M-A-G and [MG]-M[TI]-C (Figure 6). Interestingly, based on LMDIPrep results, the above last three linear peptides at the C-terminal region were predicted to interact with PDZ domain (Supplementary data S3-S4).

\subsection{Phylogenetic analysis}

For precise phylogenetic classification, the relationship of PCV4 with 7 families of CRESS DNA viruses (Rosario et al., 2012; L. Zhao et al., 2019) was inferred. The phylogenetic tree (inserted panel, Figure 7) revealed a similar tree topology between 7 families as in the previous publications (Kazlauskas et al., 2018; Krupovic et al., 2020). Based on that result, four PCV4 sequences (including E115 strain) were clustered within family Circoviridae. Using reference Rep sequences of genera Circovirus and Cyclovirus of the familyCircoviridae, all PCV4 strains were seen belonging to genusCircovirus (Figure 7). In consistency with the early classification (Tian et al., 2020; Zhang et al., 2020), this study confirmed that PCV4 is distantly related to previously known PCV1, PCV2 and PCV3.

\section{Discussion}

\subsection{The detection of PCV4}

After the first report of PCV4 in the Hunan province, China (Zhang et al., 2020), the virus was also detected in other provinces of China (Tian et al., 2020). The presence of PCV4 in different geographic locations implied the active circulation of the virus. However, a preliminary screening failed to detect PCV4 in two European countries (Franzo et al., 2020). With the detection rates of $3.28 \%$ in six out of nine provinces in Korea (Figures 1A-1B), this study provided further evidence for the prevalence of PCV4 in pigs. Combining the detection results of the first two publications (Tian et al., 2020; Zhang et al., 2020) and this report (Figure 1C), it was noteworthy to observe that PCV4 was present in pigs of all age groups and in both clinically healthy and different clinically diseased pigs. That fact was similar to PCV2 and PCV3 infections which were found in pigs with different clinical presentations (Segales, 2012; Zhai et al., 2017). Further studies are required to elucidate any association with diseases in pigs of this novel PCV4.

\subsection{The genomic characterization and genetic relationships of PCV4}

Upon detection of a novel virus in animals suffering from a certain illness, it is common to urge epidemiological and pathological studies (Faccini et al., 2017; Franzo et al., 2018). In this study, in addition to providing supportive evidence for the active circulation of PCV4, attempts were made to describe the genetic structure as well as to investigate the potential biological important proteins encoded by PCV4 which might play roles in the replication process and/or pathogenesis of PCV4 in pigs.

Of the genomic organization, the previous study described in brief that PCV4 represents a new circovirus species, having a typical origin of replication of conserved nonanucleotide stem-loop motif (Zhang et al., 2020). In this study, besides the palindrome stem-loop structure of the origin of replication in the 5'-intergenic region (Figure 2B), more genetic features were elucidated from the nucleotide sequences of E115 strain as well as three previous PCV4 strains so far. It was found that the circular genome contains several overlapping ORFs in different orientations and the existence of two intergenic regions in between genes encoding the Rep and Cap proteins (Figure 2A, Supplementary Figure S1). These features were also observed in viruses belonging to Circovirus genus ( $\mathrm{Li}$ et al., 2010). Combining the results of genetic structure analyses (Figure 2) and phylogenetic classification (Figure 7), the study agreed with the results obtained in previous publications 
(Tian et al., 2020; Zhang et al., 2020) and that E115 and other three strains of PCV4 belonged to the genus Circovirus but phylogenetically differed from three porcine circoviruses of PCV1, PCV2 and PCV3.

Of the functional analysis, this study found essential elements known so far for DNA replication of circoviruses (Figures 2B, 3, 4), such as: the NLS mediating nuclear targeting of the viral genome (Heath, Williamson, \& Rybicki, 2006), the structure at the origin of DNA replication (Cheung, 2004), endonuclease and helicase domains of Rep (Faurez, Dory, Grasland, \& Jestin, 2009; Mankertz \& Hillenbrand, 2001). Additionally, the presence of tyrosine-based $\mathrm{Y}-\mathrm{x}-\mathrm{x}-\varphi$ and $\mathrm{P}-\mathrm{x}-\mathrm{x}-\mathrm{P}$ motifs in the Cap of PCV4 (Figure 5) might be involved in the entry process of this virus into the host cell. That inference was made by evidence that the $\mathrm{Y}-\mathrm{x}-\mathrm{x}-\varphi$ were observed in a variety of viruses including SARS-CoV, HCV, EBV or SIV viruses that contribute to the viral penetration (Hraber et al., 2020; Karamichali et al., 2017; Minakshi \& Padhan, 2014). Another effect of P-x-x-P motif in Nef protein of HIV was demonstrated to interact with SH3-domains that related to the increase of virus replication (Saksela, Cheng, \& Baltimore, 1995). Lastly, in this study, several linear peptides in the putative ORF3 protein of PCV4 were observed that are likely conserved in other putative homologous proteins of viruses belonging to family Circoviridae (Figure 5). Functional analysis predicted that these peptides might interact with the PDZ domains, an important regions associated with cell signaling pathways (Lee \& Zheng, 2010). Proteins containing PDZ domains were demonstrated as target of several different viruses (James \& Roberts, 2016; Javier \& Rice, 2011). Therefore, it was possible to imply that the putative ORF3 protein of PCV4 could alter the cell signaling pathway during the virus replication. All of the above-mentioned results of genetic analyses provided not only details about genomic structure and organization of a newly described PCV4, but also supported that the virus contains essential elements for the replication process.

\section{Conclusion}

The presence of PCV4 in different geographic locations in Korea provided additional evidence for active circulation of PCV4 in pigs in different swine producing countries. The result of genetic characterizations and phylogenetic classification supported that PCV4 is an unclassified member of genus Circovirus, containing essential elements for viral replication.

\section{Funding}

This work was supported by the Korea Science and Engineering Foundation (KOSEF) grant funded by the Korea government (No. 2020R1I1A1A01054539).

\section{Ethics approval and consent to participate}

All samples used in this study were received from Boehringer Ingelheim Vetmedica Korea Ltd. according to a Nondisclosure Agreement (No. 20190037 and 20200009) of "Diagnostic Tests for Development of Domestic Livestock Industry" signed with College of Veterinary Medicine, Seoul National University. All protocols related to samples collection were approved by Seoul National University Research and Development Business Foundation (SNU-200626-2). This article does not contain any studies with alive animals performed by any of the authors.

\section{Data availability statement}

Not available

\section{Acknowledgments}

The authors would like to thank Eun Ok Kim and Jung Ah Kim for their excellent technical assistance. Also, we sincerely thank Boehringer Ingelheim Vetmedica Korea Ltd. for providing samples for this study.

\section{Conflicts of Interest}

The authors declare that there is no conflict of interest.

\section{Figure Legends}


Figure 1. The detection results of PCV4. The detection rates (positive/ total samples) were shown according to sampling year and geographic locations (A, B). Provinces having PCV4 positive farms are indicated with red color. The positive rates were also summarized by age groups (C) which the samples were sorted into, including aborted fetus, suckling ([?] 30 days), weaned (30 to 60 days), grower (60 to 90 days), finisher ([?] 90 days) and sow.

Figure 2. Genomic characterization of E115 strain. It was illustrated that E115 has a circular genome, containing 6 putative overlapping open reading frames (ORFs) oriented in different directions (A). The stem- loop structure with conserved nonanucleotide (highlighted) was observed (B). Comparison of the nonanucleotide sequences, $(\mathrm{T} / \mathrm{n}) \mathrm{A}(\mathrm{G} / \mathrm{t}) \mathrm{TATTAC}$, between PCV4 and the viruses of the genusCircovirus (C). The scissors represented the nicking position in the nonanucleotide motif.

Figure 3. Functional domains of putative replication- associated protein (Rep). E115 strain (arrow) was predicted containing two domains of endonuclease and helicase which were well conserved for different viruses of Circovirus genus (highlighted). In each motif, the capital letters indicated conserved amino acid, ' $\mathrm{x}$ ' was any amino acid and square brackets indicate the list of acceptable amino acids in the given position.

Figure 4. Prediction of nuclear localization signal (NLS) at the N-terminus of the putative Cap. This arginine-rich region was shown based on the alignment with other members of Circovirus genus. Sequence analysis revealed that the arginine-rich region of E115 strain was aligned with the basic motifs of the NLS of PCV1, PCV2 and PCV3 which were experimentally confirmed (highlights).

Figure 5. The $P-x-x-P$ and $Y-x-x-$ motifs located in the putative capsid proteins. Capsid proteins of PCV4 were detected containing several $\mathrm{P}-\mathrm{x}-\mathrm{x}-\mathrm{P}$ and $\mathrm{Y}-\mathrm{x}-\mathrm{x}-$ motifs. $\mathrm{Y}-\mathrm{x}-\mathrm{x}-$ motifs and the first $\mathrm{P}-\mathrm{x}-\mathrm{x}-\mathrm{P}$ motif were observed in other viruses belonging to genusCircovirus . On the other hand, the second $\mathrm{P}-\mathrm{x}-\mathrm{x}-\mathrm{P}$ only detected in PCV4 which were highlighted in a box. In each motif "x" denoted as any amino acid, while represented any of $\mathrm{F}$ (phenylalanine), I (isoleucine), L (leucine) or V (valine). The brackets show the conserved amino acid in given position. The number indicated the location of each motif based on the capsid protein of E115 strain.

Figure 6. Prediction of conserved regions of the putative ORF3 protein. Based on the alignment with some members of genus Circovirus, four conserved regions in the putative ORF3 protein of E115 strain (indicated as arrow) were observed. All putative ORF3s of PCV4 were shown as in dash box. The well conserved amino acids were also highlighted. In each short linear motif, the capital letters indicated conserved amino acid, ' $\mathrm{x}$ ' was any amino acid and square brackets indicate the list of acceptable amino acids in the given positions. C-terminal regions of the putative PCV4 ORF3 protein were predicted as containing PDZ binding peptides. The number showed the position corresponding in E115 strain.

Figure 7. Phylogenetic tree of circoviruses based on amino acid sequence of Rep. Inserted panel was the phylogeny inferred from a data containing seven assigned families of CRESS DNA viruses. It was observed that the newly identified PCV4 (arrows) was grouped within family Circoviridae. Focused on that family, two well supported genus of Cyclovirus and Circovirus were observed. Of which, PCV4 was within genus Circovirus, distantly related to previous known PCV1, PCV2 and PCV3 (indicated by filled circles). Numbers on branches were bootstrap support values. Bar showed number of amino acid substitutions per site.

\section{Supplementary Legends}

Supplementary data S1. List of the putative Rep sequences used for phylogenetic inference of CRESS DNA viruses

Supplementary data S2. List of the putative Rep sequences used for phylogenetic inference of family Circoviridae

Supplementary data-S3. ORF3_motif1-PDZdomain

Supplementary data-S4. ORF3_motif234-PDZdomain 
Supplementary Table S1. Summary of characteristics of the predicted ORFs of E115 strain

Supplementary Figure S1. Genomic structure and organisation of PCV4. Three completed genomic sequences of PCV4 were available from GenBank. All elements of genome were similar between isolates and with the Korean PCV4 strain shown in Figure 1A.

\section{References}

Arruda, B., Pineyro, P., Derscheid, R., Hause, B., Byers, E., Dion, K., . . . Schwartz, K. (2019). PCV3-associated disease in the United States swine herd. Emerging microbes 8 infections, 8 (1), 684-698. doi:10.1080/22221751.2019.1613176

Bailey, T. L., Boden, M., Buske, F. A., Frith, M., Grant, C. E., Clementi, L., . . . Noble, W. S. (2009). MEME SUITE: tools for motif discovery and searching. Nucleic Acids Res, 37 (Web Server issue), W202-208. doi:10.1093/nar/gkp335

Cheung, A. K. (2004). Identification of an octanucleotide motif sequence essential for viral protein, DNA, and progeny virus biosynthesis at the origin of DNA replication of porcine circovirus type 2. Virology, 324 (1), 28-36. doi:10.1016/j.virol.2004.03.037

de Castro, E., Sigrist, C. J., Gattiker, A., Bulliard, V., Langendijk-Genevaux, P. S., Gasteiger, E., . . . Hulo, N. (2006). ScanProsite: detection of PROSITE signature matches and ProRule-associated functional and structural residues in proteins.Nucleic Acids Res, 34 (Web Server issue), W362-365. doi:10.1093/nar/gkl124

Delwart, E., \& Li, L. (2012). Rapidly expanding genetic diversity and host range of the Circoviridae viral family and other Rep encoding small circular ssDNA genomes. Virus Res, 164 (1-2), 114-121. doi:10.1016/j.virusres.2011.11.021

Faccini, S., Barbieri, I., Gilioli, A., Sala, G., Gibelli, L. R., Moreno, A., . . . Nigrelli, A. (2017). Detection and genetic characterization of porcine circovirus type 3 in Italy. Transbound Emerg Dis, 64 (6), 1661-1664. doi:10.1111/tbed.12714

Faurez, F., Dory, D., Grasland, B., \& Jestin, A. (2009). Replication of porcine circoviruses. Virol J, 6 (1), 60. doi:10.1186/1743-422X-6-60

Fenaux, M., Halbur, P. G., Haqshenas, G., Royer, R., Thomas, P., Nawagitgul, P., . . . Meng, X. J. (2002). Cloned genomic DNA of type 2 porcine circovirus is infectious when injected directly into the liver and lymph nodes of pigs: characterization of clinical disease, virus distribution, and pathologic lesions. J Virol, 76 (2), 541-551. doi:10.1128/jvi.76.2.541-551.2002

Franzo, G., Legnardi, M., Hjulsager, C. K., Klaumann, F., Larsen, L. E., Segales, J., \& Drigo, M. (2018). Full-genome sequencing of porcine circovirus 3 field strains from Denmark, Italy and Spain demonstrates a high within-Europe genetic heterogeneity. Transbound Emerg Dis, 65 (3), 602-606. doi:10.1111/tbed.12836

Franzo, G., Ruiz, A., Grassi, L., Sibila, M., Drigo, M., \& Segales, J. (2020). Lack of porcine circovirus 4 genome detection in pig samples from Italy and Spain. Pathogens, 9 (6), 433. doi:10.3390/pathogens9060433

Heath, L., Williamson, A. L., \& Rybicki, E. P. (2006). The capsid protein of beak and feather disease virus binds to the viral DNA and is responsible for transporting the replication-associated protein into the nucleus. J Virol, 80 (14), 7219-7225. doi:10.1128/JVI.02559-05

Hoang, D. T., Chernomor, O., von Haeseler, A., Minh, B. Q., \& Vinh, L. S. (2018). UFBoot2: improving the ultrafast bootstrap approximation. Mol Biol Evol, 35 (2), 518-522. doi:10.1093/molbev/msx281

Hraber, P., O’Maille, P. E., Silberfarb, A., Davis-Anderson, K., Generous, N., McMahon, B. H., \& Fair, J. M. (2020). Resources to discover and use short linear motifs in viral proteins. Trends Biotechnol, 38 (1), 113-127. doi:10.1016/j.tibtech.2019.07.004 
James, C. D., \& Roberts, S. (2016). Viral interactions with PDZ domain-containing proteins-an oncogenic trait? Pathogens, 5 (1), 8. doi:10.3390/pathogens5010008

Javier, R. T., \& Rice, A. P. (2011). Emerging theme: cellular PDZ proteins as common targets of pathogenic viruses. J Virol, 85 (22), 11544-11556. doi:10.1128/JVI.05410-11

Jiang, H., Wang, D., Wang, J., Zhu, S., She, R., Ren, X., . . . Liu, J. (2019). Induction of porcine dermatitis and nephropathy syndrome in piglets by infection with porcine circovirus type 3. J Virol, 93 (4), e02045-02018. doi:10.1128/JVI.02045-18

Johnson, M., Zaretskaya, I., Raytselis, Y., Merezhuk, Y., McGinnis, S., \& Madden, T. L. (2008). NCBI BLAST: a better web interface.Nucleic Acids Res, 36 (Web Server issue), W5-9. doi:10.1093/nar/gkn201

Karamichali, E., Serti, E., Gianneli, A., Papaefthymiou, A., Kakkanas, A., Foka, P., . . . Georgopoulou, U. (2017). The unexpected function of a highly conserved YXXPhi motif in HCV core protein. Infect Genet Evol, 54 , 251-262. doi:10.1016/j.meegid.2017.07.001

Katoh, K., \& Standley, D. M. (2013). MAFFT multiple sequence alignment software version 7: improvements in performance and usability. Mol Biol Evol, 30 (4), 772-780. doi:10.1093/molbev/mst010

Kazlauskas, D., Varsani, A., \& Krupovic, M. (2018). Pervasive chimerism in the replication-associated proteins of uncultured single-stranded DNA viruses. Viruses, 10 (4), 187. doi:10.3390/v10040187

Kedkovid, R., Woonwong, Y., Arunorat, J., Sirisereewan, C., Sangpratum, N., Lumyai, M., . . - Thanawongnuwech, R. (2018). Porcine circovirus type 3 (PCV3) infection in grower pigs from a Thai farm suffering from porcine respiratory disease complex (PRDC). Vet Microbiol, 215 , 71-76. doi:10.1016/j.vetmic.2018.01.004

Kosugi, S., Hasebe, M., Tomita, M., \& Yanagawa, H. (2009). Systematic identification of cell cycle-dependent yeast nucleocytoplasmic shuttling proteins by prediction of composite motifs. Proc Natl Acad Sci US A, 106 (25), 10171-10176. doi:10.1073/pnas.0900604106

Krupovic, M., Varsani, A., Kazlauskas, D., Breitbart, M., Delwart, E., Rosario, K., . . . Koonin, E. V. (2020). Cressdnaviricota: a virus phylum unifying seven families of rep-encoding viruses with single-stranded, circular DNA genomes. J Virol, 94 (12), e00582-00520. doi:10.1128/JVI.00582-20

Lee, H. J., \& Zheng, J. J. (2010). PDZ domains and their binding partners: structure, specificity, and modification. Cell Commun Signal, 8 (1), 8. doi:10.1186/1478-811X-8-8

Li, L., Kapoor, A., Slikas, B., Bamidele, O. S., Wang, C., Shaukat, S., . . . Delwart, E. (2010). Multiple diverse circoviruses infect farm animals and are commonly found in human and chimpanzee feces. J Virol, 84 (4), 1674-1682. doi:10.1128/JVI.02109-09

Liu, Q., Tikoo, S. K., \& Babiuk, L. A. (2001). Nuclear localization of the ORF2 protein encoded by porcine circovirus type 2. Virology, 285 (1), 91-99. doi:10.1006/viro.2001.0922

Lv, Q., Guo, K., Xu, H., Wang, T., \& Zhang, Y. (2015). Identification of putative ORF5 protein of porcine circovirus type 2 and functional analysis of GFP-fused ORF5 protein. PLOS ONE, 10 (6), e0127859. doi:10.1371/journal.pone.0127859

Mankertz, A., \& Hillenbrand, B. (2001). Replication of porcine circovirus type 1 requires two proteins encoded by the viral rep gene. Virology, 279 (2), 429-438. doi:10.1006/viro.2000.0730

Meehan, B. M., McNeilly, F., Todd, D., Kennedy, S., Jewhurst, V. A., Ellis, J. A., . . . Allan, G. M. (1998). Characterization of novel circovirus DNAs associated with wasting syndromes in pigs. J Gen Virol, 79 ( Pt 9) (9), 2171-2179. doi:10.1099/0022-1317-79-9-2171 
Minakshi, R., \& Padhan, K. (2014). The YXXPhi motif within the severe acute respiratory syndrome coronavirus (SARS-CoV) 3a protein is crucial for its intracellular transport. Virol J, 11 (1), 75. doi:10.1186/1743$422 \mathrm{X}-11-75$

Minh, B. Q., Schmidt, H. A., Chernomor, O., Schrempf, D., Woodhams, M. D., von Haeseler, A., \& Lanfear, R. (2020). IQ-TREE 2: new models and efficient methods for phylogenetic inference in the genomic era. Mol Biol Evol, 37 (5), 1530-1534. doi:10.1093/molbev/msaa015

Mitchell, A. L., Attwood, T. K., Babbitt, P. C., Blum, M., Bork, P., Bridge, A., . . . Finn, R. D. (2019). InterPro in 2019: improving coverage, classification and access to protein sequence annotations. Nucleic Acids Res, 47 (D1), D351-D360. doi:10.1093/nar/gky1100

Mokili, J. L., Rohwer, F., \& Dutilh, B. E. (2012). Metagenomics and future perspectives in virus discovery. Current opinion in virology, 2 (1), 63-77. doi:10.1016/j.coviro.2011.12.004

Mou, C., Wang, M., Pan, S., \& Chen, Z. (2019). Identification of nuclear localization signals in the ORF2 protein of porcine circovirus type 3. Viruses, 11 (12), 1086. doi:10.3390/v11121086

Opriessnig, T., Meng, X. J., \& Halbur, P. G. (2007). Porcine circovirus type 2 associated disease: update on current terminology, clinical manifestations, pathogenesis, diagnosis, and intervention strategies.J Vet Diagn Invest, 19 (6), 591-615. doi:10.1177/104063870701900601

Palinski, R., Pineyro, P., Shang, P., Yuan, F., Guo, R., Fang, Y., . . . Hause, B. M. (2017). A novel porcine circovirus distantly related to known circoviruses is associated with porcine dermatitis and nephropathy syndrome and reproductive failure. J Virol, 91 (1). doi:10.1128/JVI.01879-16

Papadopoulos, J. S., \& Agarwala, R. (2007). COBALT: constraint-based alignment tool for multiple protein sequences. Bioinformatics, 23 (9), 1073-1079. doi:10.1093/bioinformatics/btm076

Rosario, K., Breitbart, M., Harrach, B., Segales, J., Delwart, E., Biagini, P., \& Varsani, A. (2017). Revisiting the taxonomy of the family Circoviridae: establishment of the genus Cyclovirus and removal of the genus Gyrovirus. Arch Virol, 162 (5), 1447-1463. doi:10.1007/s00705-017-3247-y

Rosario, K., Duffy, S., \& Breitbart, M. (2012). A field guide to eukaryotic circular single-stranded DNA viruses: insights gained from metagenomics. Arch Virol, 157 (10), 1851-1871. doi:10.1007/s00705-012-1391-y

Saksela, K., Cheng, G., \& Baltimore, D. (1995). Proline-rich (PxxP) motifs in HIV-1 Nef bind to SH3 domains of a subset of Src kinases and are required for the enhanced growth of Nef + viruses but not for down-regulation of CD4. The EMBO Journal, 14 (3), 484-491. doi:10.1002/j.1460-2075.1995.tb07024.x

Sarkar, D., Jana, T., \& Saha, S. (2018). LMDIPred: A web-server for prediction of linear peptide sequences binding to SH3, WW and PDZ domains. PLOS ONE, 13 (7), e0200430. doi:10.1371/journal.pone.0200430

Segales, J. (2012). Porcine circovirus type 2 (PCV2) infections: clinical signs, pathology and laboratory diagnosis. Virus Res, 164 (1-2), 10-19. doi:10.1016/j.virusres.2011.10.007

Shuai, J., Wei, W., Jiang, L., Li, X., Chen, N., \& Fang, W. (2008). Mapping of the nuclear localization signals in open reading frame 2 protein from porcine circovirus type 1. Acta Biochim Biophys Sin (Shanghai), 40 (1), 71-77. doi:10.1111/j.1745-7270.2008.00377.x

Sobhy, H. (2016). A review of functional motifs utilized by viruses.Proteomes, 4 (1), 3. doi: $10.3390 /$ proteomes 4010003

Tian, R. B., Zhao, Y., Cui, J. T., Zheng, H. H., Xu, T., Hou, C. Y., . . . Chen, H. Y. (2020). Molecular detection and phylogenetic analysis of porcine circovirus 4 in Henan and Shanxi provinces of China. Transbound Emerg Dis . doi:10.1111/tbed.13714

Tischer, I., Gelderblom, H., Vettermann, W., \& Koch, M. A. (1982). A very small porcine virus with circular single-stranded DNA.Nature, 295 (5844), 64-66. doi:10.1038/295064a0 
Tisza, M. J., Pastrana, D. V., Welch, N. L., Stewart, B., Peretti, A., Starrett, G. J., . . . Buck, C. B. (2020). Discovery of several thousand highly diverse circular DNA viruses. eLife, 9 , e51971. doi:10.7554/eLife.51971

Tochetto, C., Lima, D. A., Varela, A. P. M., Loiko, M. R., Paim, W. P., Scheffer, C. M., . . Roehe, P. M. (2018). Full-genome sequence of porcine circovirus type 3 recovered from serum of sows with stillbirths in Brazil. Transbound Emerg Dis, 65 (1), 5-9. doi:10.1111/tbed.12735

Wen, L., He, K., Xiao, Q., Yu, Z., Mao, A., Ni, Y., . . . Jiang, J. (2012). A novel porcine circovirus-like agent $\mathrm{P} 1$ is associated with wasting syndromes in pigs. PLOS ONE, 7 (8), e41565. doi:10.1371/journal.pone.0041565

Ye, X., Berg, M., Fossum, C., Wallgren, P., \& Blomstrom, A. L. (2018). Detection and genetic characterisation of porcine circovirus 3 from pigs in Sweden. Virus Genes, 54 (3), 466-469. doi:10.1007/s11262-018$1553-4$

Zhai, S. L., Zhou, X., Zhang, H., Hause, B. M., Lin, T., Liu, R., . . . Wang, D. (2017). Comparative epidemiology of porcine circovirus type 3 in pigs with different clinical presentations. Virol J, 14 (1), 222. doi:10.1186/s12985-017-0892-4

Zhang, H. H., Hu, W. Q., Li, J. Y., Liu, T. N., Zhou, J. Y., Opriessnig, T., \& Xiao, C. T. (2020). Novel circovirus species identified in farmed pigs designated as porcine circovirus 4, Hunan province, China.Transbound Emerg Dis, 67 (3), 1057-1061. doi:10.1111/tbed.13446

Zhao, D., Wang, X., Gao, Q., Huan, C., Wang, W., Gao, S., \& Liu, X. (2018). Retrospective survey and phylogenetic analysis of porcine circovirus type 3 in Jiangsu province, China, 2008 to 2017. Arch Virol, 163 (9), 2531-2538. doi:10.1007/s00705-018-3870-2

Zhao, L., Rosario, K., Breitbart, M., \& Duffy, S. (2019). Chapter three - Eukaryotic circular rep-encoding single-stranded DNA (CRESS DNA) viruses: ubiquitous viruses with small genomes and a diverse host range. In M. Kielian, T. C. Mettenleiter, \& M. J. Roossinck (Eds.),Advances in Virus Research (Vol. 103, pp. 71-133): Academic Press.

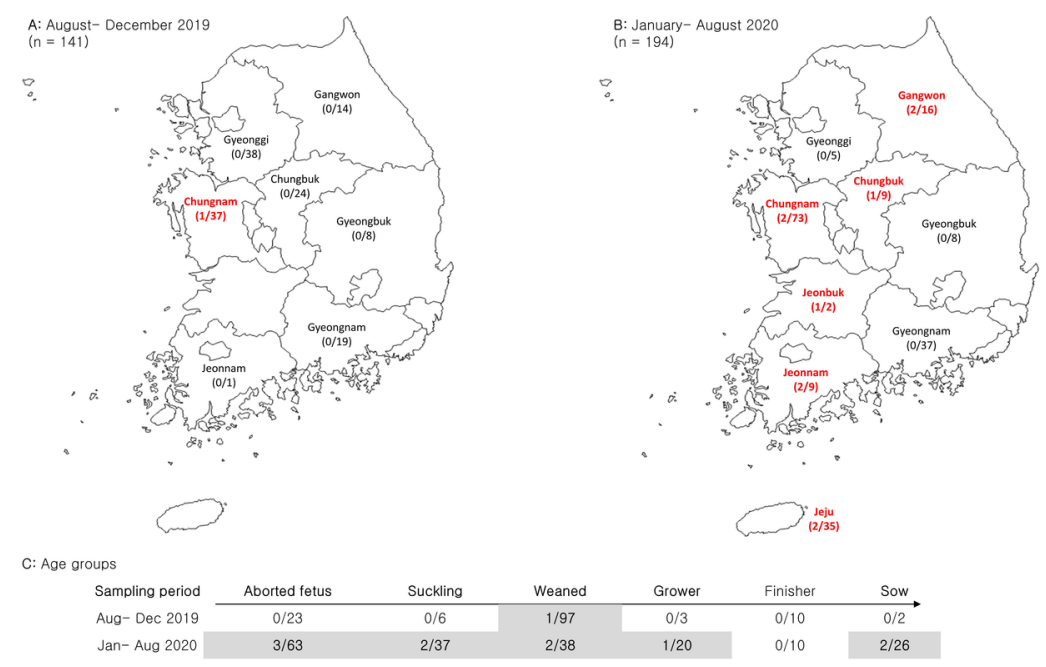



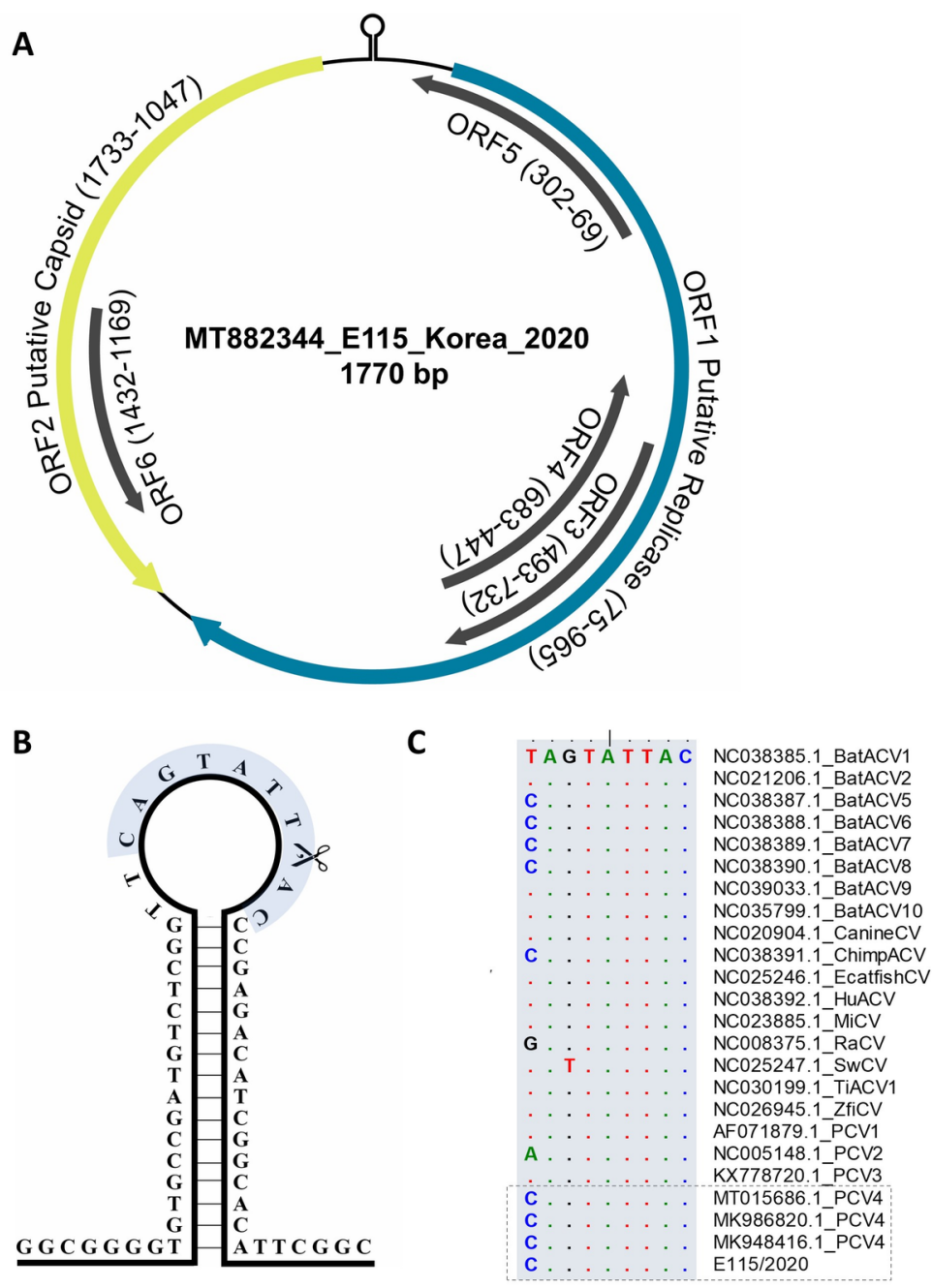

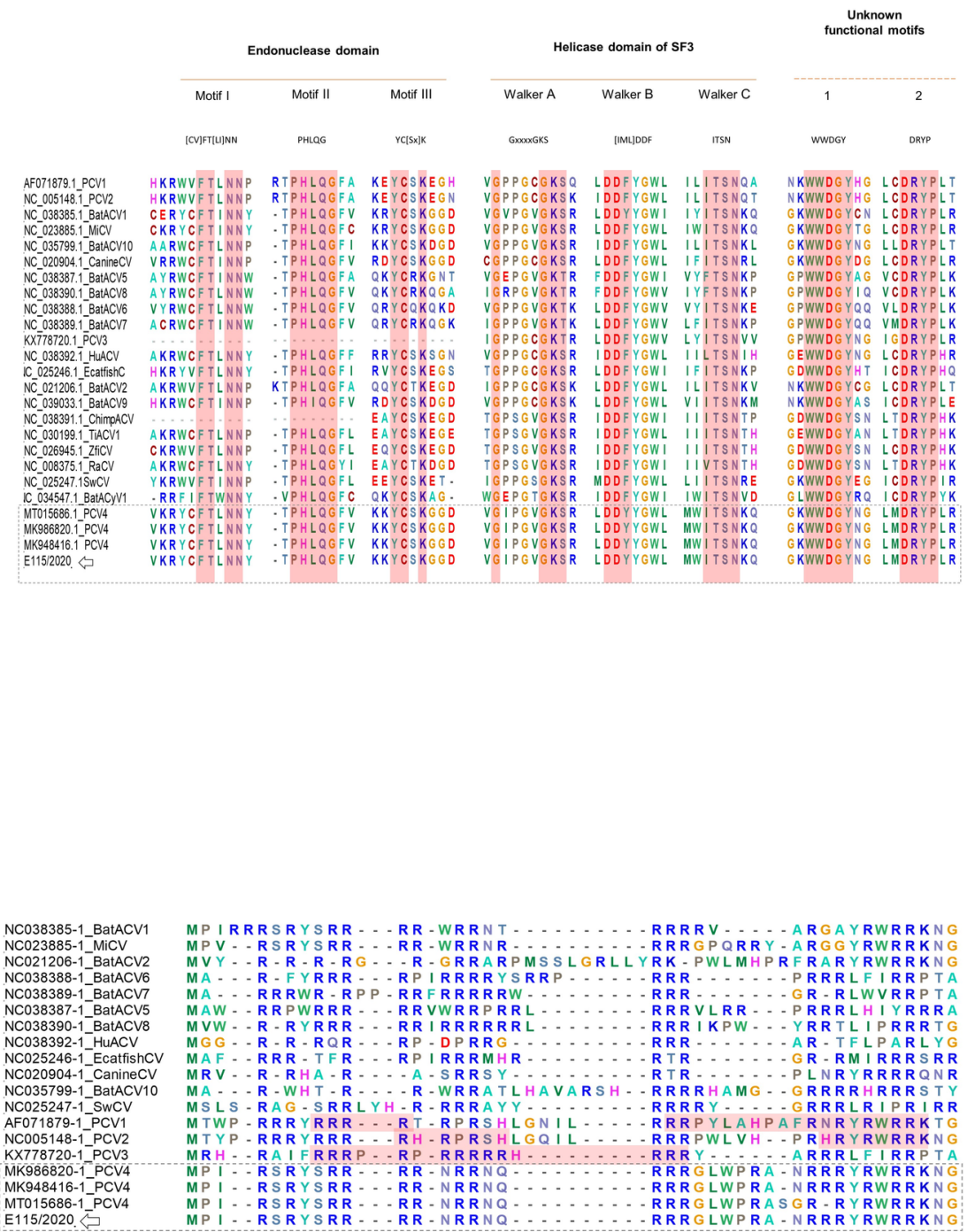


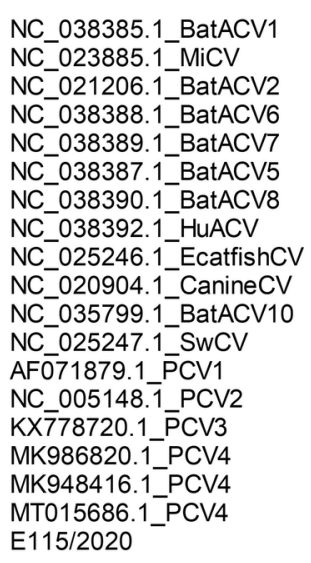

\begin{tabular}{|c|c|}
\hline$P-x-x-P$ & $1-x-x-\varphi$ \\
\hline 74 & 90 \\
\hline P A G P & Y Y R I \\
\hline$P K G P$ & Y Y R \\
\hline$P=-A$ & Y Y R \\
\hline PS - - & Y Y KM \\
\hline D T - & Y Y K \\
\hline TE - - & $Y$ \\
\hline T D - & $Y$ \\
\hline T $\mathbf{G}$ - & Y Y R \\
\hline A N - & Y Y R \\
\hline QH L P & F Y K \\
\hline P S L P & D Y N \\
\hline D R L P & Y Y M \\
\hline$P=-P$ & Y \\
\hline$P--P$ & Y \\
\hline T A - & Y Y K \\
\hline P K G P & Y Y R \\
\hline P K G P & Y Y \\
\hline P K G P & $Y Y$ \\
\hline K G P & \\
\hline
\end{tabular}

\begin{tabular}{|c|c|c|}
\hline$-\mathrm{X}$ & $-x-x-P$ & $x-x-\varphi$ \\
\hline 135 & 161 & 213 \\
\hline D P H & PDL - - - - D & Y VQF \\
\hline D & $\mathrm{H}---\cdot$ & \\
\hline & $-1=--=$ & \\
\hline & I S I GAS & \\
\hline V & $F-\cdots$ & \\
\hline$\Gamma$ & L - - - & \\
\hline & $M=--$ & \\
\hline & $\mathrm{TSA}$ & \\
\hline & F S V - - & \\
\hline Y & QD I AS F & \\
\hline S & A Q T A N & \\
\hline V & $P$ T I Q S S Q T & \\
\hline & PEL - - - & \\
\hline & P V L - - - - & \\
\hline & $P$ I LAGTTSA & \\
\hline $\mathrm{Y}$ & PQ D & \\
\hline & PQD - - & Y \\
\hline & - - & \\
\hline & $P Q D=-=-$ & \\
\hline
\end{tabular}

Motif ।

W.-AS.5[P]]-[DG]-M

NC038385.1_BatACV1

NC020904.1_CanineCV

NC025246.1_EcatfishCV NC023885.1 MiCV

AF071879.1 PCV1

NC005148.1 PCV2

PCV4_MT015686.1

PCV4_MK986820.1

PCV4_MK948416.1

PCV4_E115/2020 \&
WA S P G M:F R

A V N P G MQQTML WA SP GMYKKQL - ARP V VIGRASG WG H L G VIV KA NG WW S P D MSKRR G WWA S L D MYY KRRG INA SP DMISKRRG WA S P DMSKRR G
AARAG TAWRO-

\begin{tabular}{|c|c|c|}
\hline \multicolumn{3}{|c|}{ ind to PDZ domain } \\
\hline Motif II & Motif III & Motf IV \\
\hline 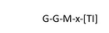 & $W-M-[T]-[(F)-M-A-G-G$ & {$[\mathrm{MG}]-\mathrm{M} \cdot[\mathrm{m}] \cdot-\mathrm{c}$} \\
\hline G G M & L M T I MDG & $M M \mid C$ \\
\hline INGGMA & WWM T SMD G & L M T C \\
\hline I G GMV & WM I F MDG & WMKC \\
\hline N G G MA & CINM I F T G G & MM T C \\
\hline is G GMD & WM I F MA G & G M I \\
\hline S G G TV & L M T F M A G & $G M I Y$ \\
\hline is G GMD & SiWM T I M A G & MM T C \\
\hline $\begin{array}{l}S \mathrm{~S} G \mathrm{M} \\
\mathrm{s}\end{array}$ & WM T I MA G & MM T C \\
\hline GMD & WM T I MAG & MM \\
\hline S G G M D & WM T I MA G & $M M I C$ \\
\hline & & \\
\hline
\end{tabular}




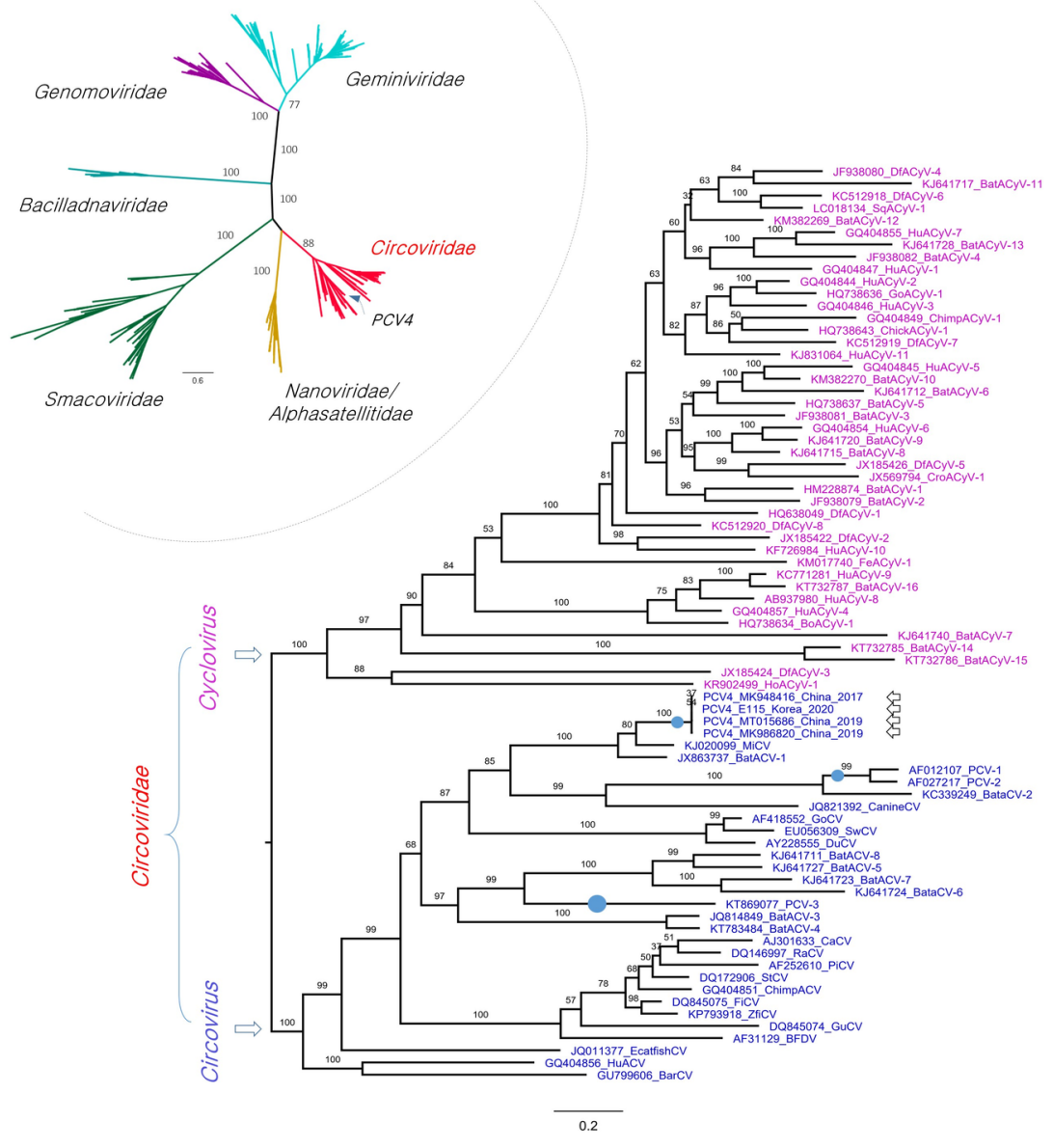

